\title{
Correlation between Intraoperative Fluid Administration and Outcomes of Pancreatoduodenectomy
}

\author{
Xuefeng Cao, ${ }^{1}$ Xixiu Wang, ${ }^{2}$ Baolei Zhao, ${ }^{1}$ Lingqun Kong, ${ }^{1}$ Lei Zhou, ${ }^{1}$ Wentao Zhu, ${ }^{1}$ \\ Xutao Lin, ${ }^{1}$ Qiangpu Chen $\left(\mathbb{D},{ }^{1}\right.$ and Xingyuan Zhang ${ }^{1}{ }^{1}$ \\ ${ }^{1}$ Department of Hepatobiliary Surgery, Binzhou Medical University Hospital, Binzhou, Shandong Province, China \\ ${ }^{2}$ Department of Cardiovascular Medicine, Binzhou Medical University Hospital, Binzhou, Shandong Province, China \\ Correspondence should be addressed to Xingyuan Zhang; byfyzxy@163.com
}

Received 1 March 2020; Revised 5 June 2020; Accepted 22 June 2020; Published 30 July 2020

Academic Editor: Tatsuya Toyokawa

Copyright (C) 2020 Xuefeng Cao et al. This is an open access article distributed under the Creative Commons Attribution License, which permits unrestricted use, distribution, and reproduction in any medium, provided the original work is properly cited.

\begin{abstract}
Background. Intraoperative fluid (IOF) administration plays an important role during major abdominal surgery although increased fluid intake can adversely influence postoperative outcomes. However, the effect of the IOF rate on the outcomes of pancreatoduodenectomy (PD) is unclear. Methods. 151 patients, who underwent PD at Binzhou Medical University Hospital between January 2010 and May 2017, were categorized into three groups according to IOF rates (ml/kg/hr): restricted $(<10, n=47)$, standard $(10-15, n=76)$, and liberal $(>15, n=28)$. Results. The overall postoperative morbidity was $56.95 \%$. The incidence of postoperative pancreatic fistula (POPF) was $11.26 \%$. The in-hospital mortality rate was $7.28 \%$ with the most common cause being grade C POPF and secondary intra-abdominal infections. The patients in the liberal group had significantly higher incidences of POPF $(25 \%)$ and respiratory complications $(21.43 \%)$. The other outcome parameters such as recovery of bowel function, hospital stay, and postoperative daily drainage were similar among the groups. Multivariable analysis confirmed the IOF rate to be most strongly associated with POPF (odds ratio: 5.195, confidence interval: 1.142-23.823, $P=0.023$ ) and respiratory complications (odds ratio: 7.302, confidence interval: $0.676-58.231, P=0.025$ ). Conclusions. The IOF rate significantly affects the incidence of POPF and respiratory complications after PD. Careful patient-oriented fluid therapy may help to prevent these complications.
\end{abstract}

\section{Introduction}

Fluid administration, especially of intraoperative fluid (IOF), is an integral part of almost any surgical procedure [1]. During operations, there are third space losses with peripheral vasodilatation due to anesthesia. Fluid loading with large amounts of intravenous (IV) fluids is done in clinical practice to expand the intravascular space and improve organ perfusion [2]. However, several studies [3-8] suggest that perioperative fluid administration (especially IOF administration) has a substantial direct impact on the outcomes in patients undergoing major abdominal surgery. Several randomized controlled trials (RCTs) and meta-analyses concerning perioperative fluid management strategies in major operations such as colorectal surgery have found that perioperative fluid restriction is associated with enhanced recovery of gastrointestinal function, and reduced complications and hospital stay [1,9-11].

Pancreatoduodenectomy (PD) is a complex procedure associated with long operative time, potentially large-volume blood loss, and high surgical morbidity. The incidence of surgical morbidities or complications is $38 \%-58 \%$-the most common being postoperative pancreatic fistula (POPF), biliary fistula, wound infection, postoperative intraperitoneal hemorrhage, and respiratory complications $[12,13]$.

During the last 60 years, postoperative mortality has markedly decreased because of improvements in surgical techniques, better perioperative management with early recognition and treatment of complications. However, morbidity after PD remains high [14, 15]. Advanced age, poor nutritional status, high preoperative serum bilirubin, and soft pancreas are important risk factors for mortality and 
complications after PD [16]. In addition to these factors, we hypothesize that perioperative fluid administration (especially IOF administration) may be another independent factor to influence the outcomes following PD. The results of previous studies on this issue have been controversial [17$22]$. Given this uncertainty, we performed this study to determine the correlation between the IOF administration and outcomes following PD.

\section{Patients and Methods}

The study was approved by the Institutional Ethics Committee of Binzhou Medical University Hospital. All the methods in the study were performed in accordance with the relevant regulations in our hospital. Informed consent of patients was not taken separately as this is a retrospective review not revealing patient identities.

2.1. Patient Selection. In this study, consecutive patients who underwent PD for benign or malignant pancreatic or periampullary diseases from January 1, 2010, to May 31, 2017, at Binzhou Medical University Hospital were included. Patients with malignant tumors who received neoadjuvant therapy or vascular resection were excluded.

2.2. Perioperative Care. All the patients received general anesthesia including drugs for premedication, induction, and maintenance of general anesthesia. As a standard practice, the fluids were used in the ratio of $3: 1$ (crystalloid: colloid). The rate of IOF was at the discretion of the anesthesiologists. Blood transfusions were given when hemoglobin levels were $<8 \mathrm{~g} / \mathrm{dl}$. Blood was given with crystalloid at a ratio of $1: 3$, and colloid at a ratio of $1: 1$. The details about IOF administration including the fluid type (crystalloid, colloid, or blood product) and the amount of fluid used were recorded.

For this study, we used the IOF rate $(\mathrm{ml} / \mathrm{kg} / \mathrm{hr})$-intraoperative infusion volume divided by the duration of the operation and the weight of the patient (instead of the simple volume)-to analyze the fluid management precisely. Similarly, POF rate $(\mathrm{ml} / \mathrm{kg} / \mathrm{hr})$ - postoperative infusion volume divided by $24 \mathrm{hr}$ and the weight of the patient-was used to analyze postoperative fluid management.

All the patients who underwent $\mathrm{PD}$ were categorized into three groups according to the IOF rate: restricted $(<10 \mathrm{ml} / \mathrm{kg} / \mathrm{hr})$, standard $(10-15 \mathrm{ml} / \mathrm{kg} / \mathrm{hr})$, and liberal $(>15 \mathrm{ml} / \mathrm{kg} / \mathrm{hr})$. The cut-off values used for these three groups were based on the current anesthesia guidelines for IOF administration (10-15 ml/kg/hr) [23-25]. The daily drainage of the first three postoperative days was expressed as drainage on POD1, POD2, and POD3. Similarly, POF rate on the first three postoperative days was expressed as the rate of POF1, POF2, and POF3.

2.3. Surgical Procedure. All the PD procedures were performed by the same surgical team. An upper midline abdominal incision was made in all cases. The procedure for PD and the digestive tract reconstruction was the same in all patients. A duct to mucosa pancreatojejunostomy was constructed using 5-0 PDS/prolene sutures. According to pancreatic duct size, the corresponding diameter of pancreatic duct stent was placed in the anastomosis. Postoperative care included intravenous antibiotics, maintenance intravenous fluids, and parenteral nutrition support.

2.4. Data Collection. Most data were collected from our clinical database including age, gender, and weight of patients; duration of operation; intraoperative infusion volume (crystalloid, colloid, and blood product); estimated intraoperative blood loss; volume of postoperative fluid (POF) administration on the first three days; postoperative outcomes such as the daily drainage on the first three postoperative days; recovery of bowel function; intensive care unit (ICU) admission; postoperative length of hospital stay; and postoperative complications. Intraoperative infusion volume, duration of operation, and estimated intraoperative blood loss data were obtained from the anesthesia records; POF volume and daily drainage of the first three postoperative days were obtained from nursing records; and surgical outcome data were obtained from our clinical records.

2.5. Definitions. ISGPS [26] criteria were used for the diagnosis and grading of POPF.

Chylous fistula was diagnosed based on the clinical manifestations and chylous test [27].

Postoperative biliary fistula was diagnosed based on the bilirubin levels in the drain fluid or on the postoperative contrast study.

Postpancreatectomy hemorrhage and delayed postoperative gastric emptying were diagnosed and graded according to the ISGPS criteria $[28,29]$.

2.6. Statistical Analysis. The continuous variables were expressed as the mean and standard deviation (SD). The continuous and categorical variables were compared using ANOVA and Chi-squared tests, respectively. The multivariate analysis was conducted by logistic regression model. All the analyses were performed with Statistical Package for Social Sciences (SPSS) version 24.0 software (IBM Co, Armonk, NY, USA). A $P$ value $<0.05$ was considered to be statistically significant.

\section{Results}

3.1. Baseline Characteristics. 168 PDs were performed during the study period; 17 patients who underwent PD combined with vascular resection were excluded. Finally, 151 patients (92 males, 59 females) with a mean age of 58.52 years were included in the study and underwent PD. The mean weight of the patients was $62.78 \mathrm{~kg}$ (range 45 to $100 \mathrm{~kg}$ ). There were $47(31.13 \%), 76(50.33 \%)$, and $28(18.54 \%)$ patients in the restricted, standard, and liberal groups, respectively. The malignant pathologies included ampullary adenocarcinoma $(n=38,25.17 \%)$, cholangiocarcinoma $(n=35,23.18 \%)$, duodenal adenocarcinoma $(n=30,19.87 \%)$, and pancreatic adenocarcinoma $(n=26,17.22 \%)$. The mean duration of operation was 6.12 hours (range 2.90 to 11.33 hours). There were no statistical differences in age, sex ratio, preoperative serum albumin (ALB) level, and body mass index (BMI) among the three groups $(P>0.05$, Table 1$)$. 
TABLE 1: Clinical characteristics of the study patients $(n=151)$, and comparison of preoperative and intraoperative characteristics among the three intravenous fluid administration rate groups.

\begin{tabular}{|c|c|c|c|c|c|}
\hline Parameter & Total $(n=151)$ & Restricted group $(n=47)$ & Standard group $(n=76)$ & Liberal group $(n=28)$ & $P$ value \\
\hline Age (years) & $59.11 \pm 10.25$ & $59.11 \pm 10.25$ & $56.91 \pm 9.76$ & $61.93 \pm 10.39$ & .072 \\
\hline Gender & & & & & .209 \\
\hline Male & $92(60.93 \%)$ & $31(65.96 \%)$ & $48(63.16 \%)$ & $13(46.43 \%)$ & \\
\hline Female & $59(39.07)$ & $16(34.04 \%)$ & $28(36.84 \%)$ & $15(53.57 \%)$ & \\
\hline Preop ALB & $37.94 \pm 2.53$ & $38.22 \pm 2.55$ & $37.65 \pm 2.60$ & $38.23 \pm 2.30$ & .388 \\
\hline BMI & $23.95 \pm 3.37$ & $23.94 \pm 3.41$ & $24.01 \pm 3.37$ & $23.80 \pm 3.42$ & .960 \\
\hline Comorbidities & $91(60.26 \%)$ & $29(61.70 \%)$ & $46(60.53 \%)$ & $16(57.14 \%)$ & .925 \\
\hline Hypertension & 53 & 17 & 27 & 8 & \\
\hline Diabetes & 25 & 9 & 11 & 5 & \\
\hline COPD & 9 & 2 & 6 & 2 & \\
\hline Others & 4 & 1 & 2 & 1 & \\
\hline Preop TBIL & $126.02 \pm 64.00$ & $135.36 \pm 64.84$ & $120.18 \pm 51.48$ & $126.19 \pm 89.34$ & .445 \\
\hline IOF volume (ml) & $4294 \pm 1046$ & $3990 \pm 870$ & $4309 \pm 1178$ & $4763 \pm 736$ & .007 \\
\hline Weight $(\mathrm{kg})$ & $62.78 \pm 8.24$ & $64.98 \pm 7.15$ & $61.87 \pm 8.96$ & $61.57 \pm 7.42$ & .087 \\
\hline Duration of operation (hr) & $6.12 \pm 1.51$ & $6.38 \pm 1.54$ & $5.98 \pm 1.59$ & $6.02 \pm 1.21$ & .345 \\
\hline IOF rate $(\mathrm{ml} / \mathrm{kg} / \mathrm{hr})$ & $11.89 \pm 3.53$ & $8.25 \pm 1.22$ & $12.01 \pm 1.42$ & $17.68 \pm 1.78$ & .000 \\
\hline Tumor type & & & & & .687 \\
\hline Malignant & $133(88.08 \%)$ & $41(87.23 \%)$ & $66(86.84 \%)$ & $26(92.86 \%)$ & \\
\hline Benign & $18(11.92 \%)$ & $6(12.77 \%)$ & $10(13.16 \%)$ & $2(7.14 \%)$ & \\
\hline
\end{tabular}

Abbreviations: ALB: albumin; BMI: body mass index; COPD: chronic obstructive pulmonary disease; TBIL: total bilirubin; IOF: intraoperative fluid.

3.2. Perioperative Fluid Administration. The IOF volume ranged from 2600 to $8100 \mathrm{ml}$. The mean IOF rate was $11.89 \mathrm{ml} / \mathrm{kg} / \mathrm{hr}$ (range 4.85 to $21.43 \mathrm{ml} / \mathrm{kg} / \mathrm{hr}$ ). Sixteen patients (10.60\%) suffered from excessive bleeding and hypotension and required blood transfusion. The mean volume of blood transfusion was $500 \mathrm{ml}$. The mean POF rate on the first three postoperative days was $2.08,2.04$, and $2.04 \mathrm{ml} / \mathrm{kg} / \mathrm{hr}$, respectively (Table 2 ).

3.3. Postoperative Outcomes. The overall postoperative morbidity was $56.95 \%$ with 55 patients suffering from at least one complication. The incidence of POPF was $11.26 \%$, while the incidence of biliary fistula was $5.96 \%$. The in-hospital mortality rate was $7.28 \%$ with $54.55 \%$ caused by grade C POPF and secondary intra-abdominal infections. The mean postoperative hospital stay was $19.78 \pm 9.79$ days. The mean duration of recovery of bowel function was $4.66 \pm 1.35$ days. The daily drain outputs on POD1-POD3 were $133 \pm 112 \mathrm{ml}$, $105 \pm 162 \mathrm{ml}$, and $72 \pm 150 \mathrm{ml}$, respectively. Details of the postoperative outcomes are listed in Table 2.

3.4. Comparison of Outcomes among the Three Groups. Intraoperative characteristics and postoperative outcomes among the three groups are compared in Table 2. The pancreatic fistula rate and respiratory complications of the liberal group patients were significantly higher than restricted group patients $(P=0.035,0.025$, respectively). There was no statistically significant difference in the other outcomes such as volume of blood transfusion, postoperative length of stay, biliary fistula, postoperative drain output, and postpancreatectomy hemorrhage among the groups $(P>0.05)$.

On multivariable analysis, in addition to plasma ALB levels and BMI of patients, IOF was also a factor significantly associated with POPF and respiratory complications. Additionally, POPF was significantly associated with respiratory complications. The multivariable analysis revealed that the IOF rate was an independent factor associated with POPF (odds ratio: 5.195, confidence interval: 1.142-23.823, $P=$ 0.023 ) and respiratory complications (odds ratio: 7.302, confidence interval: 0.676-58.231, $P=0.025$ ) (Table 3).

\section{Discussion}

In recent years, several studies have found that the IOF rate can influence postoperative outcomes after major abdominal surgery. PD is associated with significant morbidity and mortality [30]. It is the touchstone to demonstrate the relationship between IOF administration and the postoperative outcomes. However, the data on IOF administration and post PD outcomes are sparse and controversial.

In the current study, we found that the liberal fluid regimen during PD was associated with significantly higher incidence of POPF $(P=0.035)$ as well as respiratory complications $(P=0.027)$ compared to the restricted and standard fluid regimen. However, the POPF rate was similar in the three groups when BL, grade B fistula, and grade C fistula were analyzed separately because of the small number of patients in each group. Moreover, there was no statistically significant difference in the other outcomes such as 
TABLE 2: Details of intraoperative characteristics, postoperative outcome $(n=151)$, and comparison of them among the three groups.

\begin{tabular}{|c|c|c|c|c|c|}
\hline Parameter & $\begin{array}{c}\text { Total } \\
(n=151)\end{array}$ & $\begin{array}{l}\text { Restricted group } \\
\quad(n=47)\end{array}$ & $\begin{array}{l}\text { Standard group } \\
\quad(n=76)\end{array}$ & $\begin{array}{l}\text { Liberal group } \\
\quad(n=28)\end{array}$ & $\begin{array}{c}P \text { value (restricted vs. } \\
\text { liberal) }\end{array}$ \\
\hline IOF rate $(\mathrm{ml} / \mathrm{kg} / \mathrm{hr})$ & $11.89 \pm 3.53$ & $8.25 \pm 1.22$ & $12.01 \pm 1.42$ & $17.68 \pm 1.78$ & \\
\hline Estimated blood loss (ml) & $313 \pm 238$ & $330 \pm 230$ & $285 \pm 176$ & $360 \pm 364$ & .304 \\
\hline Patients transfused in PD & $16(10.60 \%)$ & $3(6.38 \%)$ & $8(10.53 \%)$ & $5(17.86 \%)$ & \\
\hline $\begin{array}{l}\text { Volume of blood transfusion } \\
(\mathrm{ml})\end{array}$ & $500 \pm 146$ & $467 \pm 115$ & $500 \pm 151$ & $520 \pm 179$ & .897 \\
\hline POF1 rate $(\mathrm{ml} / \mathrm{kg} / \mathrm{hr})$ & $2.08 \pm 0.45$ & $2.07 \pm 0.44$ & $2.08 \pm 0.46$ & $2.08 \pm 0.46$ & .980 \\
\hline POF2 rate $(\mathrm{ml} / \mathrm{kg} / \mathrm{hr})$ & $2.04 \pm 0.45$ & $2.03 \pm 0.44$ & $2.06 \pm 0.47$ & $2.02 \pm 0.43$ & .899 \\
\hline POF3 rate $(\mathrm{ml} / \mathrm{kg} / \mathrm{hr})$ & $2.04 \pm 0.44$ & $2.02 \pm 0.43$ & $2.08 \pm 0.47$ & $1.98 \pm 0.41$ & .575 \\
\hline $\begin{array}{l}\text { Postoperative length of stay } \\
\text { (days) }\end{array}$ & $19.78 \pm 9.79$ & $22.36 \pm 11.10$ & $18.82 \pm 9.66$ & $18.07 \pm 6.78$ & .088 \\
\hline $\begin{array}{l}\text { Recovery of bowel function } \\
\text { (days) }\end{array}$ & $4.66 \pm 1.35$ & $4.72 \pm 1.57$ & $4.62 \pm 1.26$ & $4.79 \pm 1.20$ & .829 \\
\hline Drainage of POD1 (ml) & $133 \pm 112$ & $112 \pm 71$ & $140 \pm 119$ & $148 \pm 144$ & .286 \\
\hline Drainage of POD2 (ml) & $105 \pm 162$ & $110 \pm 169$ & $102 \pm 150$ & $104 \pm 187$ & .962 \\
\hline Drainage of POD3 (ml) & $72 \pm 150$ & $62 \pm 108$ & $76 \pm 163$ & $76 \pm 178$ & .867 \\
\hline Complications & $86(56.95 \%)$ & & & & \\
\hline $\mathrm{POPF}^{[26]}$ & $17(11.26 \%)$ & $3(6.38 \%)$ & $7(9.21 \%)$ & $7(25 \%)$ & .035 \\
\hline $\mathrm{BL}$ & 4 & 1 & 2 & 1 & \\
\hline Grade B & 6 & 1 & 2 & 3 & \\
\hline Grade C & 7 & 1 & 3 & 3 & \\
\hline Biliary fistula & $9(5.96 \%)$ & $3(6.38 \%)$ & $4(5.26 \%)$ & $2(7.14 \%)$ & .927 \\
\hline Wound infection & $12(7.95 \%)$ & $3(6.38 \%)$ & $5(6.58 \%)$ & $4(14.29 \%)$ & .459 \\
\hline $\begin{array}{l}\text { Postpancreatectomy } \\
\text { hemorrhage }\end{array}$ & $11(7.28 \%)$ & $2(4.26 \%)$ & $6(7.89 \%)$ & $3(10.71 \%)$ & .558 \\
\hline Intra-abdominal infection & $10(6.62 \%)$ & $2(4.26 \%)$ & $5(6.58 \%)$ & $3(10.71 \%)$ & .553 \\
\hline Respiratory complication & $15(9.93 \%)$ & $1(2.13 \%)$ & $8(10.53 \%)$ & $6(21.43 \%)$ & .025 \\
\hline Pulmonary infection & $9(5.96 \%)$ & 1 & 3 & 5 & \\
\hline Pulmonary edema & $5(3.31 \%)$ & 0 & 1 & 4 & \\
\hline Pulmonary embolus & $1(0.66 \%)$ & 0 & 1 & 0 & \\
\hline Delayed gastric emptying ${ }^{[29]}$ & $5(3.31 \%)$ & $1(2.13 \%)$ & $2(2.63 \%)$ & $2(7.14 \%)$ & .456 \\
\hline Chylous fistula ${ }^{[27]}$ & $3(2.65 \%)$ & 0 & $2(2.63 \%)$ & $1(0.77 \%)$ & .485 \\
\hline Liver abscess & $2(1.32 \%)$ & $1(2.13 \%)$ & $1(1.32 \%)$ & $0(0)$ & .738 \\
\hline Others & $2(1.32 \%)$ & 0 & $1(1.32 \%)$ & $1(0.77 \%)$ & .738 \\
\hline Mortality (in-hospital) & $11(7.28 \%)$ & $2(4.26 \%)$ & $4(5.26 \%)$ & $5(17.86 \%)$ & .057 \\
\hline $\begin{array}{l}\text { POPF/intra-abdominal } \\
\text { infection }\end{array}$ & 6 & 0 & 2 & 4 & \\
\hline Hemorrhage & 3 & 1 & 1 & 1 & \\
\hline Pulmonary embolus & 1 & 0 & 1 & 0 & \\
\hline Acute renal failure & 1 & 1 & 0 & 0 & \\
\hline
\end{tabular}

Abbreviations: IOF: intraoperative fluid; POF: postoperative fluid; POD: postoperative day; POPF: postoperative pancreatic fistula; BL: biochemical leak.

postoperative hospital stay, recovery of bowel function, biliary fistula, wound infection, and postpancreatectomy hemorrhage. The overall in-hospital mortality was $7.28 \%$, and the overall postoperative morbidity was $56.95 \%$.

An earlier retrospective analysis by Lindenblatt et al. (2008) [31] revealed no significant association between the incidence of postoperative bleeding $(8.2 \%)$, wound infection (4.1\%), POPF $(9.4 \%)$, mortality $(2.0 \%)$, and the volume of
IOF administered; the authors concluded that IOF should be targeted at $10-15 \mathrm{ml} / \mathrm{kg} / \mathrm{hr}$. However, their analysis included other pancreatic resections along with PD. A study by Boland et al. [32] of 188 patients who underwent $\mathrm{PD}$ for adenocarcinoma concluded that the volume of IOC increased with the operative time, blood loss, and intraoperative blood transfusion, but did not correlate with postoperative morbidities. However, a retrospective analysis by Eng 
TABLE 3: Multivariate regression analysis: factors affecting complications.

\begin{tabular}{|c|c|c|c|c|c|c|}
\hline & \multicolumn{3}{|c|}{ Pancreatic fistula } & \multicolumn{3}{|c|}{ Respiratory complication } \\
\hline & Odds ratio & $95 \% \mathrm{CI}$ & $P$ value & Odds ratio & $95 \% \mathrm{CI}$ & $P$ value \\
\hline IOF rate $(\mathrm{ml} / \mathrm{kg} / \mathrm{hr})$ & 5.195 & $1.142-23.823$ & .023 & 7.302 & $0.676-58.231$ & .025 \\
\hline Preop ALB & 19.053 & $3.048-11.126$ & .000 & 15.967 & $1.378-118.636$ & .000 \\
\hline BMI & 18.658 & $2.892-10.301$ & .000 & 17.055 & $1.626-133.721$ & .000 \\
\hline Estimated blood loss (ml) & 0.555 & $.063-4.874$ & .596 & 0.271 & $.033-2.613$ & .602 \\
\hline Duration of operation (hr) & 1.278 & $.234-6.983$ & .777 & 0.711 & $.092-7.057$ & .399 \\
\hline POF1 rate $(\mathrm{ml} / \mathrm{kg} / \mathrm{hr})$ & 0.805 & $.50-13.067$ & .878 & 0.906 & $.101-9.431$ & .341 \\
\hline POF2 rate $(\mathrm{ml} / \mathrm{kg} / \mathrm{hr})$ & 4.812 & $.410-56.425$ & .211 & 1.925 & $.292-27.465$ & .165 \\
\hline POF3 rate $(\mathrm{ml} / \mathrm{kg} / \mathrm{hr})$ & 0.486 & $.052-4.569$ & .528 & 0.166 & $.023-2.336$ & .684 \\
\hline Benign/malignant & 0.269 & $.069-1.055$ & .060 & 1.555 & $.163-10.712$ & .212 \\
\hline Pancreatic fistula & & & & 91.384 & $10.359-901.33$ & .000 \\
\hline
\end{tabular}

Abbreviations: IOF: intraoperative fluid; POF: postoperative fluid; ALB: albumin; BMI: body mass index.

et al. [33] showed that high IOF rate was associated with worse perioperative outcomes in patients undergoing PD, especially in those with preoperative serum albumin $\leq 3.0 \mathrm{~g} / \mathrm{dl}$. Another recent study [17] demonstrated that high IOF volume $(\geq 8.2 \mathrm{ml} / \mathrm{kg} / \mathrm{hr})$ was associated with an increased incidence of POPF after PD.

The mechanism behind the occurrence of POPF is poorly understood. Recent research [34] suggests that excessive blood loss, pancreatic duct size $(<5 \mathrm{~mm})$, soft pancreatic parenchyma, and certain disease pathologies were associated with increased fistula rates. Moreover, two studies $[6,35]$ showed that excessive intravenous hydration diminishes tissue oxygenation, causes edema, and may cause poor healing of the anastomosis.

The pancreas is a solid organ and lacks distensibility. We believe that a high IOF rate promotes pancreatic and peripancreatic edema formation which hampers healing of pancreato-intestinal anastomosis leading to pancreatic fistula. In contrast, the fistula involving cholangio-intestinal anastomosis and/or gastro-intestinal anastomosis seldom occurs, because both sides of the anastomosis are cavity organs with high distensibility. Besides, the pancreatic anastomosis has a poorer blood supply than cholangio-intestinal anastomosis or gastro-intestinal anastomosis, which makes it more prone to leakage.

Unlike other studies, we demonstrated a statistically significant difference in the incidence of postoperative respiratory complications $(P=0.027)$. Most of these respiratory complications (mainly ARDS and pleural effusion) were seen secondary to grade $\mathrm{C}$ POPF and intra-abdominal collections.

However, there are several limitations of this study. First, it is a retrospective study and liable to selection bias. A prospective analysis about the relationship between perioperative fluid management and complications following PD is currently in development which will address this issue. Second, although we found that there was a correlation between IOF volume and some of the outcomes following PD, IOF administration is just a part of perioperative fluid management, and postoperative fluid administration may also play a vital role in the outcomes. Third, several other factors such as pancreatic duct diameter and pancreatic consistency were not studied which could affect the outcomes as seen in previous studies. Lastly, this was a single-center study with a small sample size.

In conclusion, IOF management during $\mathrm{PD}$ is crucial and affects the incidence of POPF and respiratory complications. A restrictive fluid therapy may help to prevent those complications. Future randomized clinical trials comparing different fluid regimens are required to validate our hypotheses.

\section{Data Availability}

The datasets generated and analyzed during the present study are available from the corresponding author on reasonable request.

\section{Ethical Approval}

The study was approved by the Institutional Ethics Committee of Binzhou Medical University Hospital, Shandong Province, China. All procedures performed in studies involving human participants were in accordance with the ethical standards of the institutional and national research committee and with the 1964 Helsinki Declaration and its later amendments or comparable ethical standards. Written informed consent of patients was not taken separately as this is a retrospective review not revealing patient identities.

\section{Conflicts of Interest}

No benefits in any form have been received or will be received from a commercial party related directly or indirectly to the subject of this article.

\section{Acknowledgments}

This research was supported by the Medical and Health Science and Technology Development Plan of Shandong Province (No. 2018WSB29012) and the Science and Technology Plan of Binzhou Medical University (No. BY2015KYQD21). 


\section{References}

[1] C. G. Byers, "Fluid therapy: options and rational selection," The Veterinary Clinics of North America. Small Animal Practice, vol. 47, no. 2, pp. 359-371, 2017.

[2] S. McGloin, "The ins and outs of fluid balance in the acutely ill patient," The British Journal of Nursing, vol. 24, no. 1, pp. 1418,2015

[3] P. S. Myles, S. Andrews, J. Nicholson, D. N. Lobo, and M. Mythen, "Contemporary approaches to perioperative IV fluid therapy," World Journal of Surgery, vol. 41, no. 10, pp. 2457-2463, 2017.

[4] P. S. Myles and R. Bellomo, "Restrictive or liberal fluid therapy for major abdominal surgery," The New England Journal of Medicine, vol. 379, no. 13, p. 1283, 2018.

[5] M. W. Manning, W. J. Dunkman, and T. E. Miller, "Perioperative fluid and hemodynamic management within an enhanced recovery pathway," Journal of Surgical Oncology, vol. 116, no. 5, pp. 592-600, 2017.

[6] B. Brandstrup, "Fluid therapy for the surgical patient," Best Practice \& Research. Clinical Anaesthesiology, vol. 20, no. 2, pp. 265-283, 2006.

[7] S. Bampoe, P. M. Odor, A. Dushianthan et al., "Perioperative administration of buffered versus non-buffered crystalloid intravenous fluid to improve outcomes following adult surgical procedures," Cochrane Database of Systematic Reviews, 2017.

[8] V. A. Bennett and M. Cecconi, "Perioperative fluid management: from physiology to improving clinical outcomes," Indian J Anaesth, vol. 61, no. 8, pp. 614-621, 2017.

[9] Y. Huang, T. C. Chua, A. J. Gill, and J. S. Samra, "Impact of perioperative fluid administration on early outcomes after pancreatoduodenectomy: A meta-analysis," Pancreatology, vol. 17, no. 3, pp. 334-341, 2017.

[10] T. Corcoran, J. Emma Joy Rhodes, S. Clarke, P. S. Myles, and K. M. Ho, "Perioperative Fluid Management Strategies in Major Surgery," Anesthesia \& Analgesia, vol. 114, no. 3, pp. 640-651, 2012.

[11] M. Sandini, S. Paiella, M. Cereda et al., "Perioperative Interstitial Fluid Expansion Predicts Major Morbidity Following Pancreatic Surgery: Appraisal by Bioimpedance Vector Analysis," Annals of Surgery, vol. 270, no. 5, pp. 923-929, 2019.

[12] M. L. DeOliveira, J. M. Winter, M. Schafer et al., "Assessment of Complications After Pancreatic Surgery," Annals of Surgery, vol. 244, no. 6, pp. 931-939, 2006.

[13] A. Z. Le Bian, D. Fuks, S. Chopinet et al., "Consequences of metabolic syndrome on postoperative outcomes after pancreaticoduodenectomy," World Journal of Gastroenterology, vol. 23, no. 17, pp. 3142-3149, 2017.

[14] A. J. Edis, P. D. Kiernan, and W. F. Taylor, "Attempted curative resection of ductal carcinoma of the pancreas: review of Mayo Clinic experience, 1951-1975," Mayo Clinic Proceedings, vol. 55, pp. 531-536, 1980.

[15] T. E. Clancy, "Surgery for pancreatic cancer," Hematology/Oncology Clinics of North America, vol. 29, no. 4, pp. 701-716, 2015.

[16] W. G. Wang, S. R. Babu, L. Wang, Y. Chen, B. L. Tian, and H. B. He, "Use of Clavien-Dindo classification in evaluating complications following pancreaticoduodenectomy in 1, 056 cases: a retrospective analysis from one single institution," Oncology Letters, vol. 16, pp. 2023-2029, 2018.
[17] S. Andrianello, G. Marchegiani, E. Bannone et al., "Clinical implications of intraoperative fluid therapy in pancreatic surgery," Journal of Gastrointestinal Surgery, vol. 22, no. 12, pp. 2072-2079, 2018.

[18] F. M. Grant, M. Protic, M. Gonen, P. Allen, and M. F. Brennan, "Intraoperative fluid management and complications following pancreatectomy," Journal of Surgical Oncology, vol. 107, no. 5, pp. 529-535, 2013.

[19] S. Wang, X. Wang, H. Dai, J. Han, N. Li, and J. Li, "The Effect of Intraoperative Fluid Volume Administration on Pancreatic Fistulas after Pancreaticoduodenectomy," Journal of Investigative Surgery, vol. 27, no. 2, pp. 88-94, 2013.

[20] G. P. Wright, T. J. Koehler, A. T. Davis, and M. H. Chung, "The drowning whipple: perioperative fluid balance and outcomes following pancreaticoduodenectomy," Journal of Surgical Oncology, vol. 110, no. 4, pp. 407-411, 2014.

[21] L. Weinberg, D. Wong, D. Karalapillai et al., "The impact of fluid intervention on complications and length of hospital stay after pancreaticoduodenectomy (Whipple's procedure)," BMC Anesthesiology, vol. 14, no. 1, 2014.

[22] R. Behman, S. Hanna, N. Coburn et al., "Impact of fluid resuscitation on major adverse events following pancreaticoduodenectomy," American Journal of Surgery, vol. 210, no. 5, pp. 896-903, 2015.

[23] M. Sendak, "Monitoring and management of perioperative fluid and electrolyte therapy," in Principles and Practice of Anesthesiology, 1st edn, pp. 863-966, Mosby-Year Book, New York, 1993.

[24] P. Perel, I. Roberts, K. Ker, and Cochrane Injuries Group, "Colloids versus crystalloids for fluid resuscitation in critically ill patients," Cochrane Database of Systematic Reviews, 2013.

[25] V. Saini, S. M. Assu, N. Bhatia, and S. Sethi, "Abdominal surgery in a patient with bullous emphysema: anesthetic concerns," Journal of Anaesthesiology Clinical Pharmacology, vol. 35, no. 3, pp. 414-415, 2019.

[26] C. Bassi, G. Marchegiani, C. Dervenis et al., "The 2016 update of the International Study Group (ISGPS) definition and grading of postoperative pancreatic fistula: 11 Years After," Surgery, vol. 161, no. 3, pp. 584-591, 2017.

[27] M. G. Besselink, L. B. van Rijssen, C. Bassi et al., "Definition and classification of chyle leak after pancreatic operation: A consensus statement by the International Study Group on Pancreatic Surgery," Surgery, vol. 161, no. 2, pp. 365-372, 2017.

[28] M. N. Wente, J. A. Veit, C. Bassi et al., "Postpancreatectomy hemorrhage (PPH): an international study group of pancreatic surgery (ISGPS) definition," Surgery, vol. 142, no. 1, pp. 20-25, 2007.

[29] M. N. Wente, C. Bassi, C. Dervenis et al., "Delayed gastric emptying (DGE) after pancreatic surgery: a suggested definition by the international study group of pancreatic surgery (ISGPS)," Surgery, vol. 142, no. 5, pp. 761-768, 2007.

[30] P. J. Lakhey, R. S. Bhandari, B. Ghimire, and M. Khakurel, "Perioperative outcomes of pancreaticoduodenectomy: Nepalese experience," World Journal of Surgery, vol. 34, no. 8, pp. 1916-1921, 2010.

[31] N. Lindenblatt, S. Park, G. Alsfasser, M. Gock, and E. Klar, "Intraoperative fluid management in pancreatic resectionsthe surgeon's view," Zentralblatt für Chirurgie, vol. 133, no. 2, pp. 168-175, 2008.

[32] M. Melis, F. Marcon, A. Masi et al., "Effect of intraoperative fluid volume on peri-operative outcomes after 
pancreaticoduodenectomy for pancreatic adenocarcinoma," Journal of Surgical Oncology, vol. 105, no. 1, pp. 81-84, 2012.

[33] O. S. Eng, J. Goswami, D. Moore et al., "Intraoperative fluid administration is associated with perioperative outcomes in pancreaticoduodenectomy: a single center retrospective analysis," Journal of Surgical Oncology, vol. 108, no. 4, pp. 242-247, 2013.

[34] M. P. Callery, W. B. Pratt, T. S. Kent, E. L. Chaikof, and C. M. Vollmer Jr., "A prospectively validated clinical risk score accurately predicts pancreatic fistula after pancreatoduodenectomy," Journal of the American College of Surgeons, vol. 216, no. 1, pp. 1-14, 2013.

[35] K. Jonsson, J. A. Jensen, W. H. Goodson III. et al., “Tissue Oxygenation, Anemia, and Perfusion in Relation to Wound Healing in Surgical Patients," Annals of Surgery, vol. 214, no. 5, pp. 605-613, 1991. 